\title{
Immunohistochemical Identification of Transforming Growth Factor $-\beta$ and Its Binding Protein in Human Gastrointestinal Carcinoma
}

\author{
Takayuki Mizoi, Haruo Ohtani, Seiki Matsuno and \\ Hiroshi Nagura \\ Department of Pathology and Surgery, Tohoku University \\ School of Medicine, Sendai 980
}

\begin{abstract}
Mizoi, T., Оhtani, H., Matsuno, S. and Nagura, H. Immunohistochemical Identification of Transforming Growth Factor- $\beta$ and Its Binding Protein in Human Gastrointestinal Carcinoma. Tohoku J. Exp. Med., 1992, 168 (2), 271-273 - We attempted to clarify the tissue localization of TGF- $\beta$ and latent TGF- $\beta$ binding protein (LTBP) in human gastrointestinal carcinomas by immunohistochemistry. The immunoreactivity for TGF- $\beta$ was observed in both carcinoma cells and stromal cells, particularly in diffuse-type gastric carcinoma. The immunoreactivity for LTBP was observed only in stromal cells. These results suggest that both carcinoma cells and stromal cells may produce TGF- $\beta$, and that only stromal cells may produce LTBP in gastrointestinal carcinoma. _- TGF- $\beta$; latent TGF- $\beta$ binding protein ; gastrointestinal carcinoma ; immunohistochemistry ; immunoelectron microscopy
\end{abstract}

TGF- $\beta$ plays an important role in fibrosis, wound healing, cell differentiation and many other biological responses. It has been reported that most scirrhous gastric carcinomas reveal higher expression of TGF- $\beta$ mRNA than normal tissues by Northern blot analysis (Yoshida et al. 1989). However, it is not clear whether cancer cells or stromal cells mainly produce TGF- $\beta$. TGF- $\beta$ is synthesized and secreted as an inactive complex including mature TGF- $\beta$ ( $25 \mathrm{kd})$, TGF- $\beta$ latency associated protein $(75 \mathrm{kd})$ and latent TGF- $\beta$ binding protein (LTBP, $125 \sim 160$ kd) (Miyazono et al. 1988; Wakefield et al. 1988). The distribution of TGF- $\beta$ complex including LTBP in human tissues has not been clarfied. To elucidate the distribution of the complex in human gastrointestinal carcinomas, we investi gated the immunolocalization of TGF- $\beta$ and LTBP in human gastrointestinal carcinoma.

\section{Materials and Methods}

We used frozen sections from surgical specimens of gastrointestinal carcinomas (gastric carcinoma: 25 cases, colorectal carcinoma: 6 cases). The specimens were rapidly frozen

Address for reprints : 1-1 Seiryomachi, Aoba-ku, Sendai 980, Japan. 
after the fixation with PLP. As the control, normal appearing gastric tissues were obtained away from carcinoma. Indirect immunoperoxidase method was applied for immunolocalization of TGF- $\beta$ and LTBP. Double immunostaining was also used to characterize the cells that were positive for LTBP. In this study we used anti-desmin as muscle-specific marker, anti-CD 68 as macrophage-specific marker and anti-EMA as epithelial cell-specific marker. Pre-embedding immunoelectron microscopy was performed to clarify the ultrastructural immunolocalizations of TGF- $\beta$ and LTBP.

The polyclonal antibody against human TGF- $\beta$ was purchased from Biosciences Co. of Texas. The polyclonal antibody against human LTBP was kindly provided by Dr. K. Miyazono (Ludwig Inst. Cancer Res., Uppsala, Sweden). The negative controls included (a) replacement of primary antibody with pre-immunizing or non-immunizing serum and (b) preabsorption with the antigen peptide.

\section{RESUlts}

In diffuse (undifferentiated) type gastric carcinoma, the immunoreactivity for TGF- $\beta$ was observed not only in carcinoma cells ( 6 of 13 cases) but also in stromal spindle-shaped cells (9 of 13 cases) (Fig. 1A). The immunoreactivity for TGF- $\beta$ was blocked by preabsorption with mature TGF- $\beta$. Few cases of intestinal (differentiated) type gastric carcinoma and colorectal carcinoma had positive stromal cells for TGF- $\beta$ ( 2 of 14 cases, 0 of 6 cases) (Table 1 ). Ultrastructurally, TGF- $\beta$ positive spindle-shaped cells were identified as fibroblasts. These results suggest that both carcinoma cells and stromal cells may produce TGF- $\beta$ particularly in diffuse type gastric carcinoma. The immunoreactivity for LTBP was observed only in the stromal cells (Fig. 1B and Table 1). This reactivity was

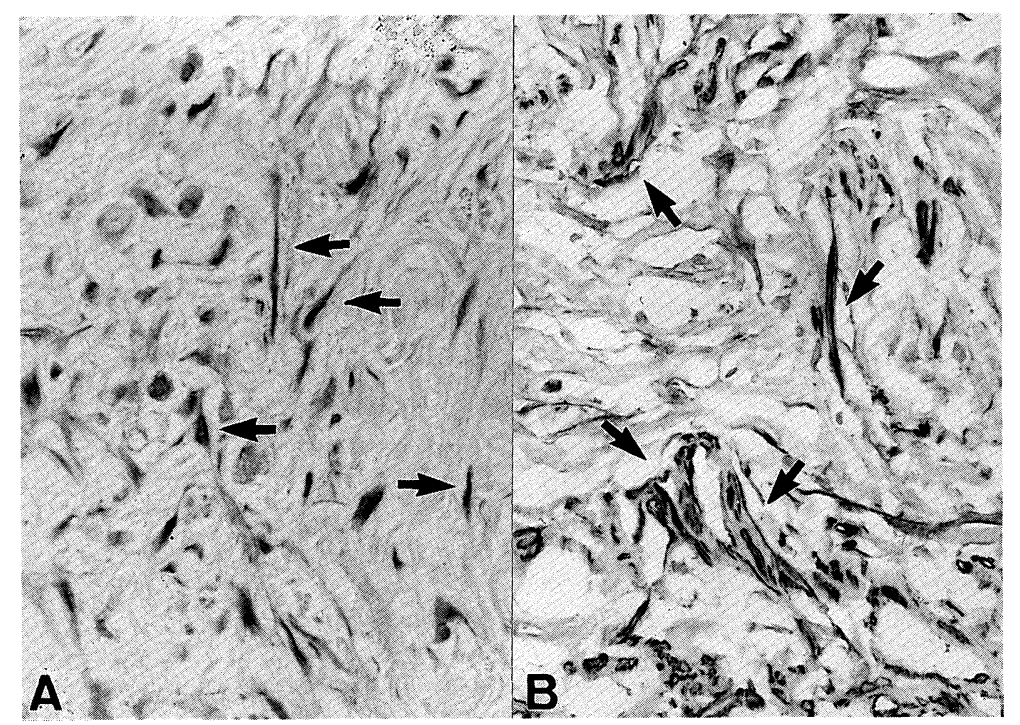

Fig. 1. Immunostaining for TGF- $\beta$ and LTBP in diffuse-type gastric carcinoma.

A : Stromal spindle-shaped cells were positive for TGF- $\beta$ (arrows).

B : Stromal spindle-shaped cells were positive for LTBP (arrows). 
TABLE 1. Immunostaining for TGF- $\beta$ and LTBP in Gastrointestinal Carcinomas (positive cases/total cases)

\begin{tabular}{ccccc}
\hline & \multicolumn{2}{c}{ TGF- $\beta$} & \multicolumn{2}{c}{ LTBP } \\
& $\mathrm{Ca}$ & $\mathrm{S}$ & $\mathrm{Ca}$ & $\mathrm{S}$ \\
\hline Gastric cancer & & & & \\
Diffuse type & $6 / 13$ & $9 / 13$ & $0 / 13$ & $12 / 13$ \\
Intestinal type & $5 / 12$ & $1 / 12$ & $0 / 12$ & $9 / 12$ \\
& & & & \\
Colorectal cancer & $0 / 6$ & $0 / 6$ & $0 / 5$ & $4 / 5$ \\
\hline
\end{tabular}

$\mathrm{Ca}$, cancer cells; S, stromal spindle-shaped cells.

completely blocked by preabsoption with the antigen. In the double immunostaining, the LTBP positive spindle-shaped cells were negative for EMA and CD68. Some spindle-shaped cells were positive for both LTBP and desmin. Ultrastructurally, immunoreactivity for LTBP was observed in rough endoplasmic reticulum of the fibroblasts and smooth muscle cells in cancer stroma. The extracellular staining for LTBP was characteristically observed around the smooth muscle cells and collagen fibers. The difference between the localization of TGF- $\beta$ and LTBP suggests a possibility that carcinoma cells may produce TGF- $\beta$ as a complex without LTBP.

\section{Acknowledgments}

We are grateful to Dr. K. Miyazono for supplying us with Ab-39 antibody and native LTBP, and for helpful discussion.

\section{References}

1) Miyazono, K., Hellman, U., Wernstedt, C. \& Heldin, C.-H. (1988) Latent high molecular weight complex of transforming growth factor $\beta 1$ : Purification from human platelets and structural characterization. J. Biol. Chem., 263, 6407-6415.

2) Wakefield, L.M., Smith, D.M., Flanders, K.C. \& Sporn, M.B. (1988) Latent transforming growth factor- $\beta$ from human platelets: A high molecular complex containing precursor sequences. J. Biol. Chem., 263, 7646-7654.

3) Yoshida, K., Yokozaki, H., Niimoto, M., Ito, H., Ito, M. \& Tahara, E. (1989) Expression of TGF- $\beta$ and procollagen type I and type III in human gastric carcinomas. Int. J. Cancer, 44, 394-398. 